BMC

Genomics

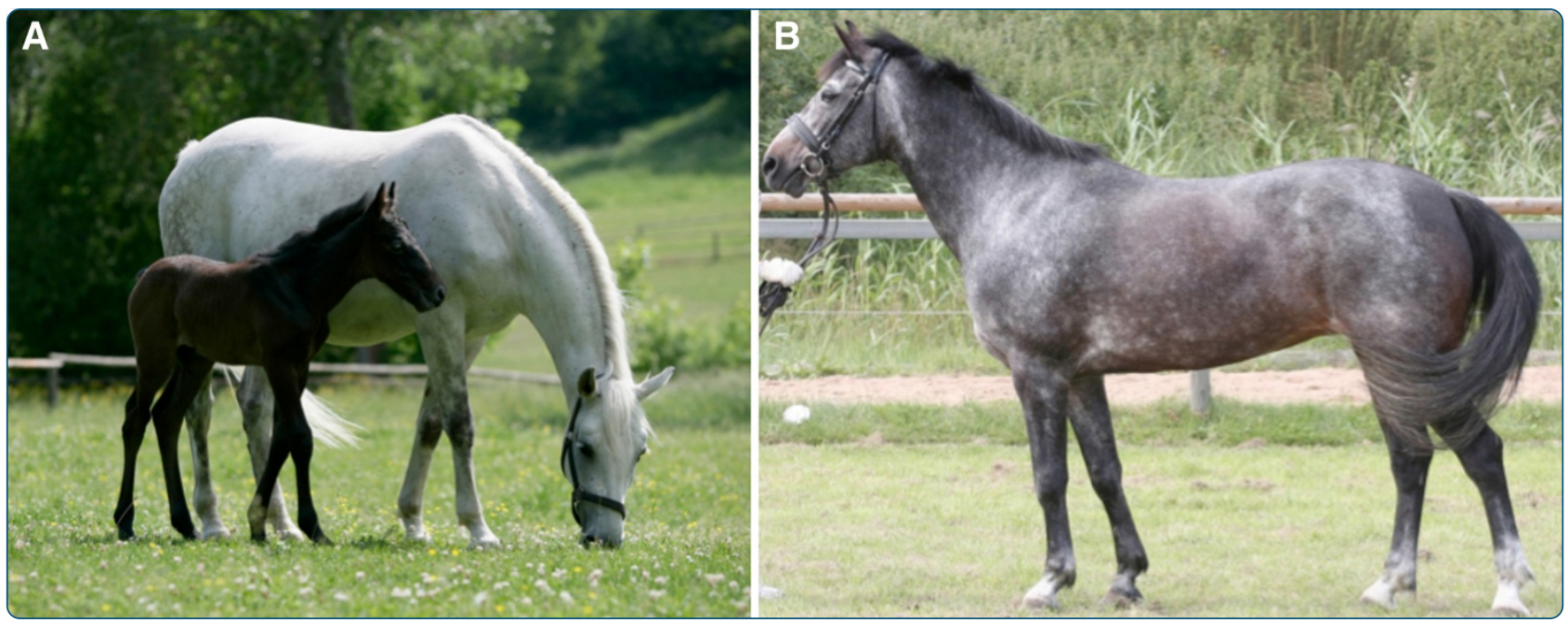

\title{
Copy number expansion of the STX17 duplication in melanoma tissue from Grey horses
}

Sundström et al. 


\title{
Copy number expansion of the STX17 duplication in melanoma tissue from Grey horses
}

\author{
Elisabeth Sundström ${ }^{1,2}$, Freyja Imsland ${ }^{2}$, Sofia Mikko ${ }^{3}$, Claire Wade ${ }^{4,5,6}$, Snaevar Sigurdsson ${ }^{4}$, \\ Gerli Rosengren Pielberg ${ }^{2}$, Anna Golovko ${ }^{2}$, Ino Curik ${ }^{7}$, Monika H Seltenhammer ${ }^{8}$, Johann Sölkner ${ }^{9}$, \\ Kerstin Lindblad-Toh ${ }^{2,4}$ and Leif Andersson ${ }^{1,2^{*}}$
}

\begin{abstract}
Background: Greying with age in horses is an autosomal dominant trait, associated with loss of hair pigmentation, melanoma and vitiligo-like depigmentation. We recently identified a $4.6 \mathrm{~kb}$ duplication in STX17 to be associated with the phenotype. The aims of this study were to investigate if the duplication in Grey horses shows copy number variation and to exclude that any other polymorphism is uniquely associated with the Grey mutation.

Results: We found little evidence for copy number expansion of the duplicated sequence in blood DNA from Grey horses. In contrast, clear evidence for copy number expansions was indicated in five out of eight tested melanoma tissues or melanoma cell lines. A tendency of a higher copy number in aggressive tumours was also found. Massively parallel resequencing of the $\sim 350 \mathrm{~kb}$ Grey haplotype did not reveal any additional mutations perfectly associated with the phenotype, confirming the duplication as the true causative mutation. We identified three SNP alleles that were present in a subset of Grey haplotypes within the $350 \mathrm{~kb}$ region that shows complete linkage disequilibrium with the causative mutation. Thus, these three nucleotide substitutions must have occurred subsequent to the duplication, consistent with our interpretation that the Grey mutation arose more than 2,000 years before present.

Conclusions: These results suggest that the mutation acts as a melanoma-driving regulatory element. The elucidation of the mechanistic features of the duplication will be of considerable interest for the characterization of these horse melanomas as well as for the field of human melanoma research.
\end{abstract}

Keywords: STX17, Melanoma, Hair greying, Copy number variation, Melanocytes

\section{Background}

Greying with age in horses is an autosomal dominant trait, associated with a gradual loss of hair pigmentation, a high incidence of melanoma and vitiligo-like depigmentation. The phenotype can be traced back through thousands of years in European and Asian culture and throughout history the white horse has been commonly used to manifest purity, power and status. Consequently, humans have favoured the phenotype in breeding over hundreds of generations. Still today, white-coated horses are preferred in many breeds all over the world,

\footnotetext{
* Correspondence: leif.andersson@imbim.uu.se

'Department of Animal Breeding and Genetics, Swedish University of Agricultural Sciences, Box 597, SE-751 24 Uppsala, Sweden

${ }^{2}$ Science for Life Laboratory Uppsala, Department of Medical Biochemistry and Microbiology, Uppsala University, Box 582, SE-751 23 Uppsala, Sweden Full list of author information is available at the end of the article
}

particularly notably in the Lipizzaner breed used in the world famous Spanish Riding School in Vienna.

Grey horses are born coloured and gradually lose their hair pigmentation (Figure 1A). Most individuals in breeds where the Grey allele is common, like Arabian, Andalusian and Lipizzaner horses, become white by the age of $6-8$ years $[1,2]$, and sometimes even earlier. The pigmentation loss only affects the hair, while the skin stays dark throughout life, indicating different cellular fates for the melanocytes in the hair follicles and in the skin. Melanomas are frequently occurring among many Grey horses, usually in the later stages of life. It is generally claimed that $70-80 \%$ of Grey horses older than 15 years have melanomas [3,4]. The primary tumours arise in the dermis of the glabrous skin, often around the eyes, genital regions or the anus, and are usually

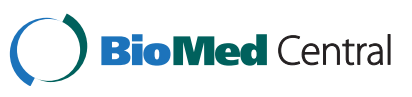




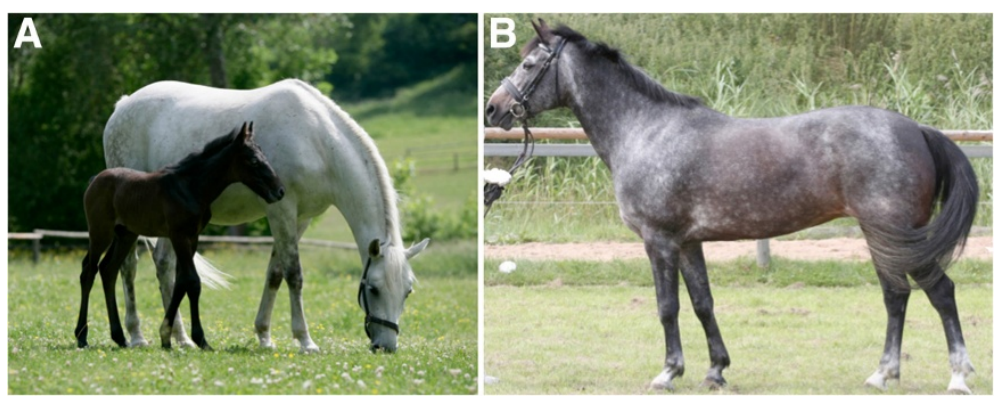

Figure 1 The Grey horse phenotype. (A) Grey horse with a dark foal. Photo: Meike Pachner. (B) A late greying Connemara horse, showing only very few signs of hair greying by the age of 14 years. Photo: Jenny Hagenblad.

benign. Internal tumours do occur but are fairly rare, and it has not been confirmed whether these tumours are true metastases or locally occurring from melanocytes residing in respective tissues. Grey horses with melanomas also develop vitiligo-like skin depigmentation, commonly seen on the muzzle and under the tail [1], and many Grey horses have pigmented speckles in their white fur, a phenotype known as flea-bitten Grey.

In 2008, we identified the mutation causing Greying with age in horses, constituting a $4.6 \mathrm{~kb}$ intronic duplication in the STX17 gene [2]. The mutation resides on a $352 \mathrm{~kb}$ haplotype showing complete linkage disequilibrium (LD) with the Grey phenotype across eight breeds. This interval was unexpectedly large since it was deduced from material including both Arabian and Icelandic horses, two divergent breeds that have been separated for a minimum of 1,000 years. As also indicated in a previous study [5], this suggests a low rate of recombination in the Grey interval. Additionally, one nonGrey haplotype identical to the Grey haplotype for all tested SNPs in the interval was suggested to represent the "ancestral" haplotype, or at least a haplotype closely related to Grey [2].

While the loss of hair pigmentation is fully dominant, the speed of greying, amount of speckling, incidence of melanomas and presence of vitiligo-like depigmentation show considerable variation among Grey horses. Horses homozygous for the mutation show a more rapid greying process and tend to become whiter than heterozygous Grey horses [2]. Homozygous individuals also show a significantly higher incidence of melanoma and vitiligo, while they almost lack the pigmented speckles present in heterozygous individuals [2]. However, there is still considerable phenotypic variation within the $G / g$ and $G / G$ genotypes both within and across breeds with respect to the manifestation of these traits. For example, some individuals in the Connemara breed carrying the Grey mutation show an extraordinarily slow rate of greying (Figure 1B). These horses show relatively few signs of greying as late as by 15 years of age, and many of them never turn completely grey. The slow rate of greying appears to be inherited within families, although the molecular reason remains unknown. Possible causes could be an alternative allele of Grey in these horses, or genetic variation elsewhere in the genome affecting the penetrance of Grey. Quantitative genetic analysis of the speed of greying in the Lipizzaner population (unpublished) indicates that a large genetic component, almost 60 percent of the phenotypic variation as regards how quickly a Grey horse become white (i.e. the speed of greying), is still unexplained.

A copy number variant (CNV) is usually defined as a region larger than $1 \mathrm{~kb}$ where copy number differences have been observed between two or more genomes [6]. Duplicated loci often show variation in copy number, which in turn may have important effects on the phenotype. This can be exemplified by the Pea-comb phenotype in chicken, where a massive and variable amplification of a duplicated sequence in intron 1 of SOX5 results in a dramatically reduced size of the comb and wattles [7], and the Dominant white phenotype in pigs, where a $450 \mathrm{~kb}$ duplication, or in some individuals triplication, in KIT causes white coat colour [8-10]. In humans, CNVs that often include large regions spanning several genes, are an important aspect of genetic variation, and have been associated with various diseases $[11,12]$. The role of CNVs in cancer is an emerging field [13]. Acquired, or somatic, copy number alterations (CNAs) in tumour DNA have for instance been identified in lung adenocarcinoma [14] and pediatric acute lymphoblastic leukaemias [15]. Copy number variants in melanocytic tumours have also been investigated, showing that melanomas and benign melanocytic nevi have different patterns of chromosomal aberrations [16].

In the present study, we have investigated whether the duplication in STX17 shows copy number variation among Grey horses. The results showed a difference in copy number of the duplication between blood and tumour DNA, with the highest copy number occurring 
in tumours classified as aggressive or derived from horses that were euthanized due to internal tumours. We also used sequence capture and next-generation sequencing to resequence the entire $352 \mathrm{~kb}$ Grey haplotype in order to exclude the possibility that other polymorphisms show as complete association to the phenotype as the STX17 duplication and may potentially contribute to the phenotype. We did not find any other mutation showing such a complete association with the Grey phenotype and thus the duplication in STX17 can be said to be well established as being causative for Greying with age.

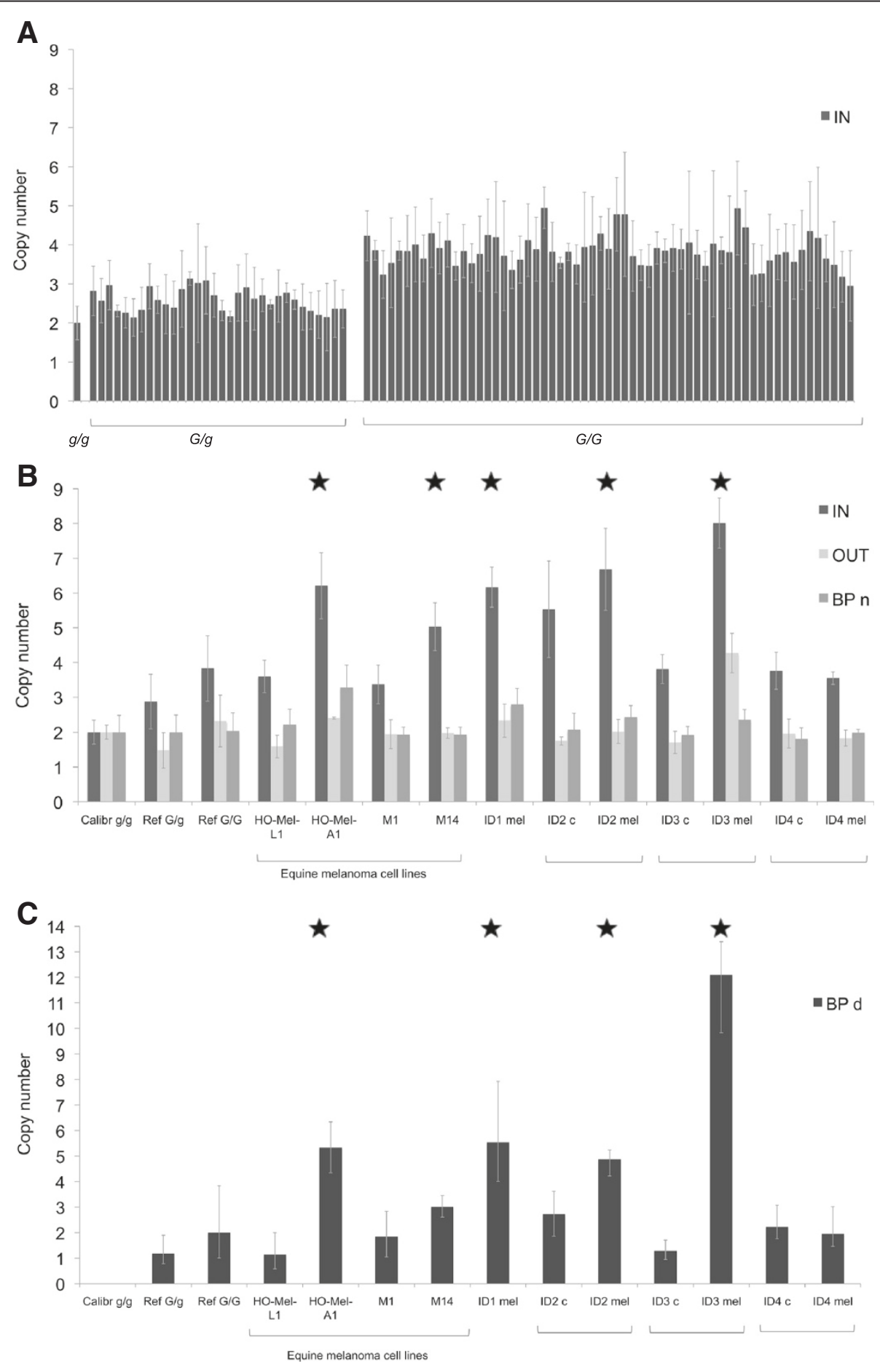

Figure 2 (See legend on next page.) 
(See figure on previous page.)

Figure 2 Copy number variation of the STX17 duplication. (A) Copy number assay for the STX17 duplication in constitutional DNA from 94 Grey Lipizzaner horses and one calibrator sample with a known copy number of 2. Mean copy number $G / g=2.58, G / G=3.84$, SEM (standard error of the mean) $\mathrm{G} / \mathrm{g}=0.05, \mathrm{G} / \mathrm{G}=0.05$. (B) Copy number assay for the STX17 duplication in constitutional DNA and/or melanoma DNA (c $=$ constitutional DNA, mel = melanoma DNA) using three different probes; IN = inside the duplicated sequence, OUT = outside the duplicated sequence and BP $\mathrm{n}=$ the border between the 5'flanking sequence and the 5'end of the duplicated sequence. The sample denoted 'Calibr' is a $\mathrm{g} / \mathrm{g}$ individual with a known copy number of 2, used as a calibrator in the analysis. Constitutional DNA from one $G / g$ and one $G / G$ horse was tested in the assay and the results are shown as a reference for the copy number expected from each genotype. Brackets surround the equine melanoma cell lines or the paired constitutional and melanoma DNA samples. Error bars represent the copy number range from the CopyCallerm ${ }^{\text {TM }}$ Software analysis of quadruplicates in each assay. Samples showing a copy number expansion are marked with an asterisk. (C) Copy number assay for the STX17 duplication breakpoint in constitutional DNA and/or melanoma DNA (c = constitutional DNA, mel = melanoma DNA) using the probe BP $d=$ over the duplication breakpoint. The $G / G$ reference sample was used as a calibrator in the analysis. Error bars represent the copy number range from the CopyCaller ${ }^{\mathrm{TM}}$ Software analysis of quadruplicates in each assay.

\section{Results}

\section{Amplification of the duplication frequently occurs in tumour DNA but not in germline}

Ninety-four homo- and heterozygous Grey Lipizzaner horses were tested for the copy number of the duplicated sequence in constitutional DNA using a TaqMan copy number assay. The results showed the expected clustering of copy number according to zygosity for Grey, and the clear separation of the Grey genotype groups did not indicate a frequent variation in copy number (Figure 2A). Analysis of tumour DNA from three horses, of which two were euthanized due to numerous internal melanomas (Table 1), and in two equine melanoma cell lines originating from aggressive tumours (Ho-Mel-A1, M14), revealed a striking expansion of the duplicated sequence, estimated to 5-8 copies (marked by an asterisk in Figure 2B).

Three different probes were initially used in the TaqMan assay (Figure 2B); $\boldsymbol{B P} \boldsymbol{n}$ located at the 5 ' border of the duplicated sequence, which is expected to be single copy if the expansion only involves the $4.6 \mathrm{~kb}$ tandem duplication; IN located within the duplicated sequence; and $\boldsymbol{O U T}$ located $1.5 \mathrm{~kb}$ downstream of the duplication, also expected to be a single copy if the expansion only involves the tandem duplication. All samples, except one, showed a normal copy number of 2.0 for both the probe at the 5 'border of the duplication (BP $\mathrm{n}$ ) and the probe outside (OUT) the duplication (Figure 2B and Additional file 1), implying that the higher copy number in tumours represents a specific amplification of the duplicated sequence rather than amplifications of a larger genomic region as often observed in tumour DNA. Only one tumour DNA sample, from individual ID3, showed a slightly higher copy number for one of the flanking probes (Figure 2B). When the region was sequenced by Sanger sequencing, no polymorphism could be detected in the probe site that would explain a lower binding affinity of the TaqMan probe, and hereby

Table 1 Samples used in the TaqMan Copy Number Assay for the Grey locus

\begin{tabular}{|c|c|c|c|c|}
\hline ID & Grey & Type $^{2}$ & Breed & Comments \\
\hline Calibrator & $g / g$ & C_DNA & Arabian & Calibrator in analysis \\
\hline Ref & $G / g$ & C_DNA & Thoroughbred & Genotype reference \\
\hline Ref & $G / G$ & C_DNA & Lipizzaner & Genotype reference \\
\hline SP1 & $G / g^{l}$ & C_DNA & Connemara & Late greying phenotype \\
\hline SP2 & $G / g^{l}$ & C_DNA & Connemara & Late greying phenotype \\
\hline SP3 & $G / g^{l}$ & C_DNA & Connemara & Late greying phenotype \\
\hline Ho-Mel-L1 & $\mathrm{G} / \mathrm{g}$ & Equine melanoma cell line & Lipizzaner & Normal growth \\
\hline Ho-Mel-A1 & $\mathrm{G} / \mathrm{g}$ & Equine melanoma cell line & Arabian & Fast growth \\
\hline M1 & $\mathrm{G} / \mathrm{g}$ & Equine melanoma cell line & Irish warmblood & Normal growth \\
\hline M14 & $G / G$ & Equine melanoma cell line & Andalusian & Fast growth \\
\hline ID1 & $\mathrm{G} / \mathrm{g}$ & tumour DNA & Thoroughbred & Euthanized due to melanoma tumours \\
\hline ID2 & $G / G$ & C_DNA + tumour DNA & Shagya Arabian & Euthanized due to melanoma tumours \\
\hline ID3 & $\mathrm{G} / \mathrm{g}$ & C_DNA + tumour DNA & Swedish warmblood & Euthanized due to melanoma tumours \\
\hline ID4 & $\mathrm{G} / \mathrm{g}$ & C_DNA + tumour DNA & Connemara & Cremello coloured $^{3}$, euthanized due to laminitis, very small melanomas \\
\hline
\end{tabular}

\footnotetext{
${ }^{1}$ Not genotyped with Grey long-range PCR assay due to fragmented DNA; inferred genotype from copy number assay.
}

${ }^{2}$ C_DNA = constitutional genomic DNA from blood or hair samples.

${ }^{3}$ Cremello horses are homozygous for a missense mutation D153N in SLC45A2 (MATP) and show a severe dilution of pigmentation in hair, skin and eye [25]. 
a false score in the analysis. To further corroborate the interpretation that the higher copy number in some tumours is caused by a local amplification of the entire tandem duplication we added a fourth TaqMan probe (BP d; Figure 2C) located exactly at the unique breakpoint between the two tandem copies on Grey chromosomes, this probe is expected to be present in $n-1$ copies if there is a local amplification of the entire duplicated sequence. The results for four out of the five tumour samples were fully consistent with a local amplification of the duplicated sequence (Figure 2C).

One non-aggressive tumour and constitutional DNA from the same individual (ID4), showed an equal copy number in both tumour and constitutional DNA, close to the expected three copies for a heterozygous Grey horse. DNA from two non-aggressive horse melanoma cell lines (Ho-Mel-L1, M1) also yielded a copy number of 3.0, in accordance with their Grey genotype determined by a diagnostic long-range PCR test.

The late greying Connemara horses showed the expected heterozygote copy number of three for the duplicated sequence, one for the duplication breakpoint and two copies of the flanking sequences (Additional file 2), verifying that these horses do indeed carry the Grey duplication.

The Grey duplication is confirmed by coverage analysis of targeted resequencing

Two individuals were chosen for resequencing of the $352 \mathrm{~kb}$ region surrounding the Grey locus, one Lipizzaner horse homozygous for Grey and one non-Grey chestnut Arabian horse carrying the closely related haplotype, a haplotype identical to Grey for all tested polymorphisms except for the duplication [2]. This experimental design was chosen to minimize the number of sequence polymorphisms identified as uniquely associated with Grey in this limited sample of resequenced horses but being non-causative. In addition, five other non-Grey horses were included in the experiment.

The read depth varied considerably between the samples, with good to exceptionally good coverage obtained for the homozygous Grey Lipizzaner, the non-Grey
Arabian and three other non-Grey horses, with two non-Grey samples showing adequate coverage for SNP calling, but not as impressive as for the rest (Table 2). This heterogeneity can in all likelihood be attributed to differences in the efficiency of enrichment between the samples (data not shown). The duplication associated with the Grey phenotype was readily observed when read depth was examined for the Grey individual across the whole resequenced region (Figure 3A).

SNP calling was performed on alignments to the repeat-masked reference sequence (Table 2). We identified more than $750 \mathrm{SNPs}$ in the region, in average 2 SNPs/kb, but the homozygous Grey horse carried a unique allele not present in the non-Grey horses at only 15 loci. The Grey homozygous Lipizzaner horse was scored as heterozygous at six of these loci, but Sangerbased resequencing revealed that all these putative heterozygous positions, except one at position 6,587,140 bp, were false positives. For 7 of the 10 remaining loci, the Grey homozygous Lipizzaner horse was either heterozygous or homozygous for the non-reference allele whereas the six non-Grey horses were homozygous for the non-reference allele at the remaining three SNPs (Figure 3B). The thoroughbred mare, named Twilight, from which the genome sequence was derived, is heterozygous at the Grey locus [17]. Thus, we postulated that the seven SNPs unique to the Grey Lipizzaner horse are in an area derived from her non-Grey allele, whereas the three non-Grey SNPs are in an area where the genome sequence is derived from Twilight's Grey allele.

\section{The duplication is the only polymorphism uniquely associated with the Grey phenotype}

The 10 SNPs uniquely associated with the Grey homozygote in the resequencing analysis were further investigated by Sanger sequencing of three additional horses; Twilight, the heterozygous horse used to generate the reference sequence, a second homozygous Grey Lipizzaner horse and a second $g / g$ non-Grey animal carrying a haplotype closely related to Grey. Two loci were excluded because the allele was also detected on a nonGrey haplotype and we failed to resequence one of these

Table 2 Statistics for alignment of sequence reads from the $352 \mathrm{~kb}$ region associated with the Grey allele

\begin{tabular}{|c|c|c|c|c|c|c|c|c|}
\hline & & \multicolumn{7}{|c|}{ Breed } \\
\hline & & Lipizzaner & Arabian & Noriker & Icelandic & Knabstrupper & Appaloosa & Quarter Horse \\
\hline \multicolumn{2}{|c|}{ Grey genotype } & $G / G$ & $g / g$ & $g / g$ & $g / g$ & $g / g$ & $g / g$ & $g / g$ \\
\hline Reads & Av. cover & 96 & 52 & 7 & 102 & 29 & 10 & 101 \\
\hline \multirow[t]{3}{*}{ SNPS } & Homozygous & 101 & 103 & 133 & 422 & 67 & 18 & 100 \\
\hline & Heterozygous & 6 & 13 & 6 & 3 & 247 & 47 & 145 \\
\hline & Total & 107 & 116 & 139 & 425 & 314 & 65 & 245 \\
\hline
\end{tabular}

Data from eight horses representing seven different breeds were compared with the horse genome reference sequence (Sep. 2007 Broad/EquCab2 assembly). SNP calling was performed based on alignments with the horse reference sequence. 


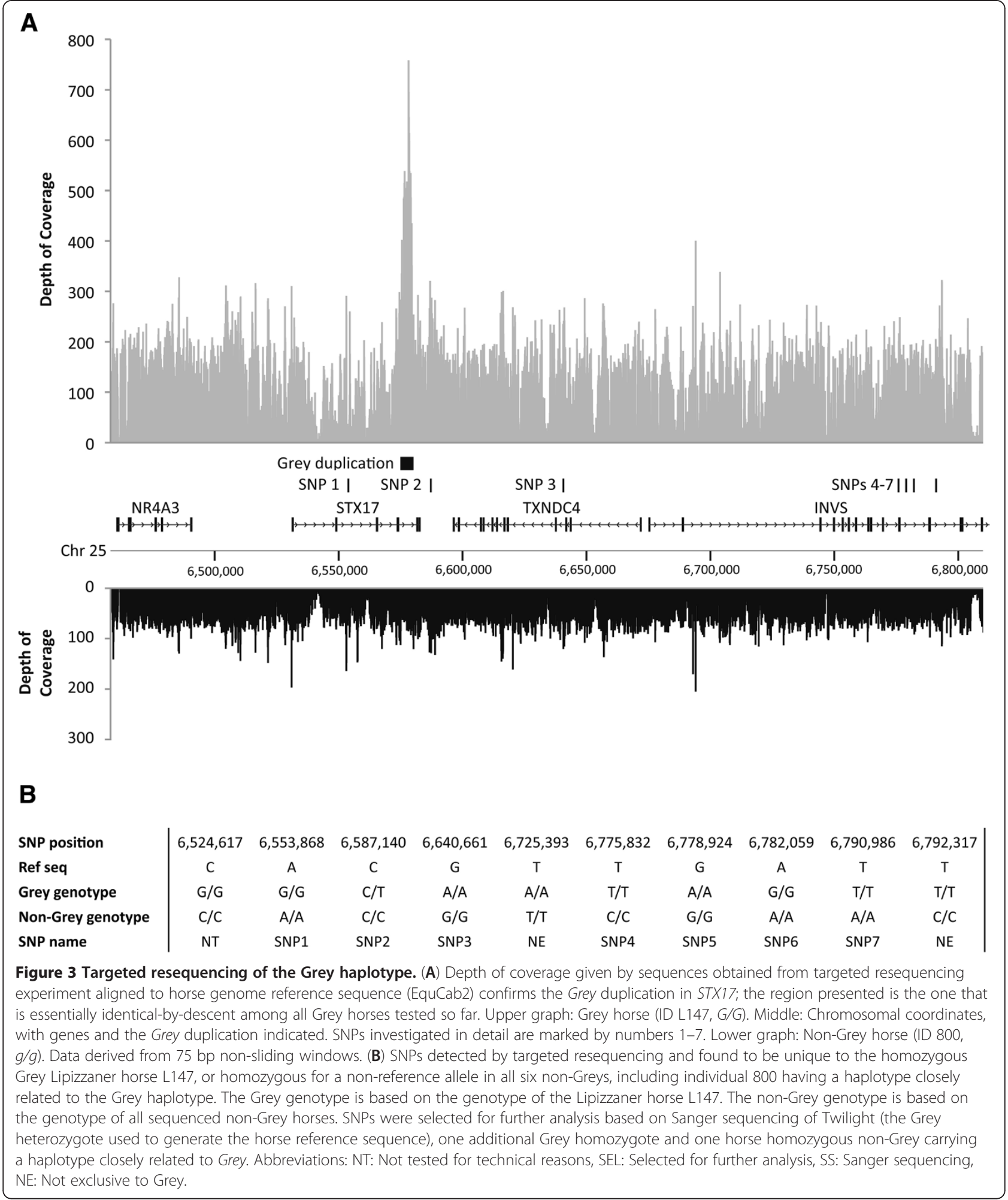

SNPs (Figure 3B). This left seven loci that were selected for further analysis.

TaqMan SNP assays were successfully designed for five of the remaining SNPs (SNP1, SNP2, SNP3, SNP4 and
SNP6; Figure 3B and Table 3) and these were genotyped in 357 Grey and non-Grey horses from 8 different breeds to reveal their association pattern to the Grey phenotype. The genotyping assays for SNP5 and SNP7 
Table 3 TaqMan genotyping of SNPs in the Grey region

\begin{tabular}{|c|c|c|c|c|c|c|c|c|c|c|c|}
\hline \multirow[b]{2}{*}{ ID } & \multirow[b]{2}{*}{ Breed } & \multirow[b]{2}{*}{ Grey } & \multicolumn{9}{|c|}{ Polymorphism $^{1}$} \\
\hline & & & SNPO & SNP1 & Dup & SNP2 & SNP3 & SNP4 & SNP5 & SNP6 & SNP7 \\
\hline 231 & Arabian & $g / g$ & & A & - & C & G & C & & A & \\
\hline 446 & Thoroughbred & $g / g$ & & A & - & C & G & C & & A & \\
\hline 1107 & New Forest Pony & $g / g$ & & A & - & C & G & $C$ & & A & \\
\hline 1268 & Icelandic & $g / g$ & & A & - & C & G & C & & A & \\
\hline 2857 & Connemara & $g / g$ & & A & - & C & G & C & & A & \\
\hline 5772 & Shetland & $g / g$ & & A & - & C & G & C & & A & \\
\hline S062 & Lipizzaner & $g / g$ & & A & - & C & G & C & & A & \\
\hline 6605 & Shetland & $g / g$ & & A & - & C & G & $\mathrm{T} / \mathrm{C}$ & $A / G$ & $\mathrm{G} / \mathrm{A}$ & A \\
\hline 1270 & Icelandic & $g / g$ & & A & - & C & - & $\mathrm{T} / \mathrm{C}$ & $\mathrm{A} / \mathrm{G}$ & $\mathrm{G} / \mathrm{A}$ & A \\
\hline 435 & Arabian & $g / g$ & & A & - & $\mathrm{C} / T^{*}$ & G & C & & A & \\
\hline 456 & Arabian: Anc & $g / g$ & & A & - & $\mathrm{C} / \mathrm{T}$ & G & C & & A & \\
\hline 800 & Arabian: Anc & $g / g$ & & A & - & $\mathrm{C} / \mathrm{T}$ & G & C & A & A & A \\
\hline 6972 & Welsh & $g / g$ & & A & - & $\mathrm{C} / \mathrm{T}$ & G & C & & A & \\
\hline D011 & Lipizzaner & $g / g$ & & A & - & $\mathrm{C} / T^{*}$ & G & C & & A & \\
\hline S046 & Lipizzaner & $g / g$ & & A & - & $\mathrm{C} / \mathrm{T}$ & G & C & & A & \\
\hline 6942 & New Forest Pony & $G / g$ & & A & $+/-$ & C & G & C & G & A & A \\
\hline S057 & Lipizzaner & $G / g$ & & A & $+/-$ & C & G & C & G & A & A \\
\hline 899 & Arabian & $G / g$ & & A & $+/-$ & C & $A / G$ & $\mathrm{~T} / \mathrm{C}$ & $A / G$ & $A$ or $G / A$ & $\mathrm{~A} / \mathrm{T}$ \\
\hline 222 & Arabian & $G / g$ & & A & $+/-$ & C & $A / G$ & $\mathrm{~T} / \mathrm{C}$ & & $\mathrm{G} / \mathrm{A}$ & \\
\hline 5726 & Connemara & $G / g$ & & A & $+/-$ & C & $\mathrm{A} / \mathrm{G}$ & $\mathrm{T} / \mathrm{C}$ & & $\mathrm{G} / \mathrm{A}$ & \\
\hline 6591 & Connemara & $G / g$ & & A & $+/-$ & C & $A / G$ & $\mathrm{~T} / \mathrm{C}$ & & $\mathrm{G} / \mathrm{A}$ & \\
\hline D081 & Lipizzaner & $G / g$ & & A & $+/-$ & C & $\mathrm{A} / \mathrm{G}$ & $\mathrm{T} / \mathrm{C}$ & & $\mathrm{G} / \mathrm{A}$ & \\
\hline D076 & Lipizzaner & $G / g$ & & $A / G$ & $+/-$ & C & $A / G$ & $\mathrm{~T} / \mathrm{C}$ & & $\mathrm{G} / \mathrm{A}$ & \\
\hline 213 & Arabian & $G / g$ & & A & $+/-$ & $\mathrm{C} / \mathrm{T}$ & $\mathrm{A} / \mathrm{G}$ & $\mathrm{T} / \mathrm{C}$ & & $\mathrm{G} / \mathrm{A}$ & \\
\hline D091 & Lipizzaner & $G / g$ & & A & $+/-$ & $\mathrm{C} / \mathrm{T}$ & $A / G$ & $\mathrm{~T} / \mathrm{C}$ & & $\mathrm{G} / \mathrm{A}$ & \\
\hline D007 & Lipizzaner & $G / g$ & & $\mathrm{~A} / \mathrm{G}$ & $+/-$ & $\mathrm{C} / \mathrm{T}$ & $A / G$ & $\mathrm{~T} / \mathrm{C}$ & & $\mathrm{G} / \mathrm{A}$ & \\
\hline D010 & Lipizzaner & $\mathrm{G} / \mathrm{g}$ & & $\mathrm{A} / \mathrm{G}$ & $+/-$ & $C / T^{*}$ & $\mathrm{~A} / \mathrm{G}$ & $\mathrm{T} / \mathrm{C}$ & & $\mathrm{G} / \mathrm{A}$ & \\
\hline 678 & Arabian & $G / g$ & & A & $+/-$ & $\mathrm{C} / \mathrm{T}$ & G & $\mathrm{T} / \mathrm{C}$ & & $\mathrm{G} / \mathrm{A}$ & \\
\hline D004 & Lipizzaner & $G / g$ & & A & $+/-$ & $\mathrm{C} / \mathrm{T}$ & G & $\mathrm{T} / \mathrm{C}$ & & $\mathrm{G} / \mathrm{A}$ & \\
\hline 452 & Thoroughbred & $\mathrm{G} / \mathrm{g}$ & & A & $+/-$ & C & G & $\mathrm{T} / \mathrm{C}$ & & $\mathrm{G} / \mathrm{A}$ & \\
\hline 3156 & Lipizzaner & $G / g$ & & A & $+/-$ & C & G & $\mathrm{T} / \mathrm{C}$ & & $\mathrm{G} / \mathrm{A}$ & \\
\hline 6577 & Welsh & $G / g$ & & A & $+/-$ & C & G & $\mathrm{T} / \mathrm{C}$ & & $\mathrm{G} / \mathrm{A}$ & \\
\hline 6611 & Shetland & $G / g$ & & A & $+/-$ & C & G & $\mathrm{T} / \mathrm{C}$ & & $\mathrm{G} / \mathrm{A}$ & \\
\hline SP1 & Connemara:Sp & $G / g$ & & A & $+/-$ & C & G & $\mathrm{T} / \mathrm{C}$ & & $\mathrm{G} / \mathrm{A}$ & \\
\hline SP2 & Connemara:Sp & $G / g$ & & A & $+/-$ & C & G & $\mathrm{T} / \mathrm{C}$ & & $\mathrm{G} / \mathrm{A}$ & \\
\hline SP3 & Connemara:Sp & $G / g$ & & A & $+/-$ & C & G & $\mathrm{T} / \mathrm{C}$ & & $\mathrm{G} / \mathrm{A}$ & \\
\hline SP4 & Connemara:Sp & $G / g$ & & - & $+/-$ & C & G & $\mathrm{T} / \mathrm{C}$ & & $\mathrm{G} / \mathrm{A}$ & \\
\hline D192 & Lipizzaner & $\mathrm{G} / \mathrm{g}$ & & $\mathrm{A} / \mathrm{G}$ & $+/-$ & C & G & $\mathrm{T} / \mathrm{C}$ & & $\mathrm{G} / \mathrm{A}$ & \\
\hline 1271 & Icelandic & $G / g$ & & A & $+/-$ & C & G & $\mathrm{T}$ & A & G & $\mathrm{A} / \mathrm{T}$ \\
\hline P023 & Lipizzaner & $G / g$ & $\mathrm{~A} / \mathrm{T}$ & $\mathrm{A} / \mathrm{G}$ & $+/-$ & $C$ & $A / G$ & $\mathrm{~T} / \mathrm{C}$ & & $\mathrm{G} / \mathrm{A}$ & \\
\hline S065 & Lipizzaner & $G / g$ & $\mathrm{~A} / \mathrm{T}$ & $\mathrm{A} / \mathrm{G}$ & $+/-$ & $C$ & - & $\mathrm{T} / \mathrm{C}$ & & $\mathrm{G} / \mathrm{A}$ & \\
\hline P038 & Lipizzaner & $\mathrm{G} / \mathrm{g}$ & $\mathrm{A} / \mathrm{T}$ & $\mathrm{A} / \mathrm{G}$ & $+/-$ & $C$ & $\mathrm{~A} / \mathrm{G}$ & $\mathrm{T} / \mathrm{C}$ & & $\mathrm{G} / \mathrm{A}$ & \\
\hline
\end{tabular}


Table 3 TaqMan genotyping of SNPs in the Grey region (Continued)

\begin{tabular}{|c|c|c|c|c|c|c|c|c|c|}
\hline L034 & Lipizzaner & $\mathrm{G} / \mathrm{g}$ & $A / T$ & $\mathrm{~A} / \mathrm{G}$ & $+/-$ & C & $\mathrm{A} / \mathrm{G}$ & - & $\mathrm{G} / \mathrm{A}$ \\
\hline P076 & Lipizzaner & $\mathrm{G} / \mathrm{g}$ & $A / T$ & $\mathrm{~A} / \mathrm{G}$ & $+/-$ & C & $\mathrm{A} / \mathrm{G}$ & $\mathrm{T} / \mathrm{C}$ & $\mathrm{G} / \mathrm{A}$ \\
\hline L040 & Lipizzaner & $\mathrm{G} / \mathrm{g}$ & $\mathrm{A} / \mathrm{T}$ & $\mathrm{A} / \mathrm{G}$ & $+/-$ & $\mathrm{C} / \mathrm{T}$ & $\mathrm{A} / \mathrm{G}$ & $\mathrm{T} / \mathrm{C}$ & $\mathrm{G} / \mathrm{A}$ \\
\hline L049 & Lipizzaner & $G / g$ & $\mathrm{~A} / \mathrm{T}$ & $\mathrm{A} / \mathrm{G}$ & $+/-$ & $C / T$ & $\mathrm{~A} / \mathrm{G}$ & $\mathrm{T} / \mathrm{C}$ & $\mathrm{G} / \mathrm{A}$ \\
\hline D007 & Lipizzaner & $\mathrm{G} / \mathrm{g}$ & $A / T$ & $\mathrm{~A} / \mathrm{G}$ & $+/-$ & $\mathrm{C} / \mathrm{T}$ & $\mathrm{A} / \mathrm{G}$ & $\mathrm{T} / \mathrm{C}$ & $\mathrm{G} / \mathrm{A}$ \\
\hline 230 & Arabian & $G / G$ & & A & $+/+$ & $C$ & A & $\mathrm{T}$ & G \\
\hline L010 & Lipizzaner & $G / G$ & & A & $+/+$ & $C$ & A & $\mathrm{T}$ & G \\
\hline D080 & Lipizzaner & $G / G$ & & $\mathrm{~A} / \mathrm{G}$ & $+/+$ & C & A & $\mathrm{T}$ & G \\
\hline D006 & Lipizzaner & $G / G$ & & G & $+/+$ & $C$ & A & $\mathrm{T}$ & G \\
\hline 399 & Arabian & $G / G$ & & A & $+/+$ & $\mathrm{C} / \mathrm{T}$ & A & $\mathrm{T}$ & G \\
\hline 857 & Arabian & $G / G$ & & A & $+/+$ & $C / T^{*}$ & A & $\mathrm{T}$ & G \\
\hline L004 & Lipizzaner & $G / G$ & & A & $+/+$ & $\mathrm{C} / \mathrm{T}$ & A & $\mathrm{T}$ & G \\
\hline D089 & Lipizzaner & $G / G$ & & $\mathrm{~A} / \mathrm{G}$ & $+/+$ & $C / T$ & A & $\mathrm{T}$ & G \\
\hline D098 & Lipizzaner & $G / G$ & & G & $+/+$ & $\mathrm{C} / \mathrm{T}$ & A & $\mathrm{T}$ & G \\
\hline 6210 & Lipizzaner & $G / G$ & & A & $+/+$ & $C$ & $\mathrm{~A} / \mathrm{G}$ & $\mathrm{T}$ & G \\
\hline D009 & Lipizzaner & $G / G$ & & $\mathrm{~A} / \mathrm{G}$ & $+/+$ & C & $A / G$ & $\mathrm{~T}$ & G \\
\hline D079 & Lipizzaner & $G / G$ & & A & $+/+$ & $C / T$ & $\mathrm{~A} / \mathrm{G}$ & $\mathrm{T}$ & G \\
\hline P086 & Lipizzaner & $G / G$ & & A & $+/+$ & $C / T^{*}$ & $\mathrm{~A} / \mathrm{G}$ & $\mathrm{T}$ & G \\
\hline D003 & Lipizzaner & $G / G$ & & $\mathrm{~A} / \mathrm{G}$ & $+/+$ & $\mathrm{C} / \mathrm{T}$ & $\mathrm{A} / \mathrm{G}$ & $\mathrm{T}$ & G \\
\hline L053 & Lipizzaner & $G / G$ & & $\mathrm{~A} / \mathrm{G}$ & $+/+$ & $C / T^{*}$ & $\mathrm{~A} / \mathrm{G}$ & $\mathrm{T}$ & G \\
\hline L024 & Lipizzaner & $G / G$ & & A & $+/+$ & C & G & $\mathrm{T}$ & G \\
\hline 6580 & Welsh & $G / G$ & & A & $+/+$ & $\mathrm{C} / \mathrm{T}$ & G & $\mathrm{T}$ & G \\
\hline D102 & Lipizzaner & $G / G$ & & A & +/+ & $C / T$ & G & $\mathrm{T}$ & G \\
\hline L090 & Lipizzaner & $G / G$ & & A & $+/+$ & $C / T^{*}$ & G & $\mathrm{T}$ & G \\
\hline
\end{tabular}

Results from TaqMan genotyping of selected SNPs located in the Grey region in 357 non-Grey and Grey horses from 8 different breeds, including the late-greying Connemara horses (Connemara:Sp) and Arabian horses (Arabian: Anc) carrying a non-Grey haplotype that is ancestral or closely related to the Grey haplotype. The table shows representative genotypes selected from all breeds. For SNP2, individuals with a strong signal for the variant T allele (C/T ratio equal 1$)$ are marked with an asterisk. SNP0 SNP5 and SNP7 were genotyped by Sanger sequencing only in key individuals. SNP0 is at nucleotide position 6,553,858 in STX17. ${ }^{1}$ SNP1 is at nucleotide position 6,553,868 in STX17; Dup is the $4.6 \mathrm{~kb}$ duplication in STX17 causing Grey; SNP2 is at nucleotide position 6,587,140 in STX17; SNP3 is at nucleotide position 6,640,661 in TXNDC4; SNP4 is at nucleotide position 6,775,832 in INVS; SNP5 is at nucleotide position 6,778,924 in INVS; SNP6 is at nucleotide position $6,782,059$ in INVS; SNP7 is at nucleotide position 6,790,986 in INVS.

failed and those two SNPs were therefore genotyped in key individuals using Sanger sequencing (Table 3).

The non-reference alleles at SNP1 and SNP3 were only found in a fraction of Grey individuals (Table 3), which means that these SNPs must have occurred subsequent to the Grey mutation. SNP1 is located in a vertebrate conserved element of approximately 20 bp (UCSC Genome Browser; Human Feb. 2009 GRCh37/hg19 Assembly), with a high regulatory potential score (UCSC Genome Browser; Human Mar. 2006 NCBI36/hg18 Assembly), while SNP3 is not. Possibly, due to its location in a conserved element, SNP1 could be functionally important, but this was not further investigated in the present study. For SNP1, a fourth, distinct separate genotype cluster was detected in the TaqMan analysis. By sequencing the amplicon for the individuals in this cluster, a SNP in the TaqMan probe site was detected, resulting in a lower $G$-allele signal in the assay, thereby creating a fourth cluster. Since the $G$ nucleotide is associated with Grey, this analysis revealed a third confirmed SNP at position 6,553,858 bp, (SNP0 in Table 3) which must have occurred on the Grey haplotype subsequent to the Grey mutation event.

SNP4 and SNP6 showed a nearly perfect association with the Grey genotype, but with the exception of two non-Grey and four heterozygous Grey individuals (Table 3). For these individuals the SNP4, SNP6 and the Grey duplication statuses were confirmed by Sanger sequencing or long-range PCR, respectively. Based on the results, these two SNPs are located just outside the haplotype block showing complete LD with the Grey mutation. Similarly, Sanger sequencing of SNP5 and SNP7, located in the vicinity of SNP6, showed that neither one was in complete LD with Grey.

In conclusion, this effort to resequence the entire $352 \mathrm{~kb}$ region associated with Grey and then further 
genotyping a set of candidate SNPs with strong associations, has provided extensive support for our previous conclusion that the $4.6 \mathrm{~kb}$ duplication in STX17 is the causative mutation for Grey. We did not identify any other sequence polymorphism uniquely associated with Grey across breeds, where the genotype distribution perfectly matched the Grey phenotype.

\section{SNP2 is duplicated in the horse genome}

Surprisingly, SNP2 did not show any significant linkage disequilibrium to Grey at all, in contrast to all other previously analyzed sequence polymorphisms located in the Grey critical interval [2]. Thus, this SNP cannot be involved in determining the Grey phenotype. In TaqMan analysis of this SNP, situated in the 3'UTR of STX17, two clearly distinct genotype clusters of individuals that appeared as heterozygous $C / T$ were detected, one cluster with a slightly higher $T$ signal. This additional cluster contained non-Grey, heterozygous Grey and homozygous Grey individuals, a finding which shows that this polymorphism shows no LD with Grey (Table 3). Sanger sequencing confirmed a heterozygous SNP genotype, and also confirmed the higher signal from the $T$-allele in some individuals (data not shown). Furthermore, no individuals homozygous for the $T$-allele were identified in the TaqMan assay, indicating that SNP2 could not be in Hardy-Weinberg equilibrium; the expected number of horses homozygous for the $T$-allele in this material was eight. We hypothesized that this anomalous result could be due to this SNP representing a second transposed copy present somewhere else in the genome. This was confirmed by a PCR-based approach used in order to determine the size of the transposed fragment. We investigated this by amplifying fragments 1 to $5 \mathrm{~kb}$ flanking the SNP, followed by SNP genotyping by Sanger sequencing. Since the $T$ allele was only detected when the flanking primer was located not more than $1 \mathrm{~kb}$ upstream or downstream of the polymorphic nucleotide, we could estimate the size of the transposed fragment to approximately $2 \mathrm{~kb}$ and it does not overlap the Grey duplication. Three additional nucleotide positions differing between the STX17 3'UTR sequence and the transposed fragment were identified (data not shown). However, we were not able to reach out into unique sequences flanking the transposed fragment by chromosome walking, which would have allowed us to determine the exact genomic position of the second copy of this sequence. An alternative way would be to map the location of this SNP with classical linkage analysis.

In conclusion, our interpretation of this anomalous SNP is that it represents a transposed copy in which the nucleotide corresponding to the $\mathrm{C}$ nucleotide at position $6,587,140$ bp in STX17 3'UTR has been replaced with a $\mathrm{T}$. Animals scored as $\mathrm{CC}$ in this assay either lack the transposed copy or carry a transposed copy with a $\mathrm{C}$ at this position. The two clusters with different $\mathrm{C}: \mathrm{T}$ ratios represent horses carrying one or two copies of the transposed sequence with the $\mathrm{T}$ nucleotide. The fact that this sequence is located elsewhere in the genome explains the complete lack of linkage disequilibrium with the Grey allele (Table 3).

\section{Discussion}

Tandem duplications are notoriously unstable and often show copy number variation [18]. In this study we have explored the possibility that the $4.6 \mathrm{~kb}$ duplication causing Greying with age in horses exhibits copy number variation in constitutional and/or tumour DNA. We show that there is a difference in copy number of the Grey duplication between constitutional DNA and tumour DNA, with the highest copy number found in tumours classified as aggressive or derived from horses euthanized possibly due to numerous internal tumours. When screening copy number expansion in constitutional DNA, we found only one horse out of about 90 with an estimated copy number of 5 or higher, whereas the corresponding figure for tumour DNA was 5 out of $8\left(\mathrm{P}=5 \times 10^{-6}\right.$; Fisher's exact test $)$. The elevated copy number in tumour DNA compared with constitutional DNA constitutes the first set of proof that the duplication in STX17 not only predisposes to melanoma development, but that copy number expansion of the duplicated sequence may be a driving force in melanoma development. The three horses showing a higher copy number in melanoma tissue than expected from their zygosity for Grey, were all euthanized possibly due to what might be internal melanoma metastases (ID1, ID2, ID3; Table 1, Figure 2B). mRNA from the tumour samples ID1 and ID2 were used for expression analysis in our previous study [19] and both samples had upregulated expression of STX17 and NR4A3 consistent with our interpretation that the copy number expansion detected in the present study may be relevant for tumour development. The melanoma cell lines showing a higher copy number than expected from the Grey genotype, Ho-Mel-A1 (Seltenhammer et al., in preparation) and M14 [20], have both been characterized as malignant, with M14 showing a high expression of the proliferating cell nuclear antigen PCNA [20], previously correlated with aggressive behaviour of human cutaneous malignant melanoma [19].

The present study has provided strong genetic support for our previous conclusion that the $4.6 \mathrm{~kb}$ duplication in intron 6 of STX17 causes Greying with age [19]. Firstly, the copy number expansion seen in tumours suggests that it may promote tumour progression. Secondly, our careful resequencing strategy of the $352 \mathrm{~kb}$ region showing complete association with Grey failed to identify 
any other sequence polymorphisms than the duplication that showed a perfect concordance with the phenotype. Furthermore, in a recent study we provided strong support for the functional significance of the duplication [21]. We demonstrate using transfection experiments and transgenic zebrafish that the duplicated sequence contains a melanocyte-specific regulatory element that is MITF (microphtalmia-associated transcription factor)-dependent. The data showed that the duplication transforms a weak enhancer element to a strong melanocyte-specific enhancer [21]. Thus, it is possible that further amplifications of the duplicated sequence, as observed in tumour samples in the present study, promote tumour development.

The great majority of sequence polymorphisms present in domestic animals predate domestication [22]. Thus, most SNP alleles associated with Grey can be expected to be shared with non-Grey haplotypes as demonstrated in this study. However, we identified three nucleotide polymorphisms that were only found in a subset of Grey horses despite the fact that they were located within the region where all sequence polymorphisms show complete LD with Grey. Thus, these sequence polymorphisms must have arisen subsequent to the Grey mutation. This result is fully consistent with the presumed long history of the Grey duplication, with an estimated age older than 2,000 years [2]. This assumption is based on the fact that all tested Grey horses representing breeds from all over the world share the same mutation that must have occurred early during horse domestication to become as widespread as it is. Furthermore, there is abundant written records 2,000 years old and older indicating the occurrence of white horses.

There is a considerable variation in how fast greying proceeds in Grey horses, and in our previous study we demonstrated that Grey homozygotes grey faster than Grey heterozygotes [2]. In this study, we have shown that several late greying Connemara horses have a copy number of three for the STX17 duplication, as expected for a heterozygous Grey horse (Additional file 2). Additionally, for the investigated SNPs, they proved to have the same haplotype as Grey horses from other breeds. This shows that the late greying phenotype in these horses is caused by the same allele as in other Grey horses, whereas there must be other modifying genetic variation in the vicinity of the Grey mutation or elsewhere in the genome.

A surprising finding in this study was that one of the SNPs corresponding to the nucleotide position $6,587,140$ bp on horse chromosome 25 , i.e. in the region showing complete LD with Grey, did not show any signs of LD with Grey. We showed that this anomaly occurs because this segment is duplicated in the horse genome and that the polymorphism is derived from the other genomic location explaining the lack of LD with Grey. The location of this second copy was not revealed in the present study but it should be straightforward to search for its location using next-generation sequence data in particular since the transposed fragment is only about $2 \mathrm{~kb}$ in size. It is possible that the presence of transposed copies of duplicated sequences is an explanation for other similar cases of one or more non-associated SNPs within an ocean of strongly associated SNPs.

\section{Conclusions}

In this paper we show that a $4.6 \mathrm{~kb}$ duplication in Syn$\operatorname{taxin} 17$, associated with the Grey phenotype in horses, does not only predispose to melanoma development, as previously shown, but that a copy number expansion of the duplication may be a driving force for melanoma development. The extensive genetic analysis, including resequencing of the $352 \mathrm{~kb}$ Grey haplotype, confirms that the $4.6 \mathrm{~kb}$ duplication is the causative mutation for this phenotype as no other polymorphism showed a complete concordance with the phenotype. The elucidation of the mechanistic features of the duplication will be of considerable interest for the characterization of these horse melanomas as well as for the field of human melanoma research.

\section{Methods}

\section{Duplication copy number variation analysis by qPCR (TaqMan)}

Constitutional DNA was prepared from blood or skin, and tumour DNA was prepared from melanoma cell cultures or melanomas (Table 1) using the DNeasy Blood \& Tissue kit (Qiagen, Valencia, CA, USA) according to manufacturer's protocol. Since the high melanin content in the melanomas can interfere with subsequent applications, the tumour DNA was purified with Chroma Spin TE-1000 columns (Clontech Laboratories Inc., Mountain View, CA, USA) as described in the standard protocol. DNA from the late greying Connemara horses was prepared either from hair by incubating 5-7 hair roots for $60 \mathrm{~min}$ at $56^{\circ} \mathrm{C}$, in a total volume of $100 \mu \mathrm{l} \mathrm{5 \%}$ chelex, containing $140 \mu \mathrm{g}$ proteinase $\mathrm{K}$, or from $100-200 \mu \mathrm{l}$ of blood using the E.Z.N.A. ${ }^{\circledR}$ SQ Blood DNA Kit (OMEGA Bio-Tech Inc., Norcross, GA, USA).

Assays for testing the potential copy number variation of the STX17 duplication and the reference gene assays were designed in PrimerExpress (Applied Biosystems, Foster City, CA, USA). Probes were designed inside and outside the duplicated sequence, and also spanning the 5 ' flanking sequence and the 5'end of the duplicated sequence and over the duplication breakpoint (Additional file 3). For each region of interest, four replicate CT values were obtained per sample with FAM $^{\mathrm{sm}}$ labelled probes for the specific regions and $\mathrm{VIC}^{\circledR}$ labelled probes 
in the reference gene assays. Two reference gene assays, RNaseP and TERT, were tested according to the recommendations by Applied Biosystems, but only the RNaseP assay was used in the analysis since TERT showed signs of amplification or deletion in several samples, as could be expected in cancer cells [23]. TaqMan Copy Number Assays were performed according to the manufacturer's instructions (Applied Biosystems) and using an ABI7900HT instrument. The results were analyzed in CopyCaller $^{\text {mix }}$ Software v1.0 (Applied Biosystems). The samples used in the assays consisted of blood DNA from one non-Grey calibrator sample, 32 heterozygous and 62 homozygous Lipizzaner horses, hair DNA from three late greying Connemara horses SP1, SP2 and SP3 (Additional file 2); tumour DNA from four equine melanoma cell lines M1, M14 [20], Ho-Mel-A1 and HO-Mel-L1 (Seltenhammer et al., in preparation), tumour DNA from one heterozygous Grey individual: ID1, and paired constitutional and tumour DNA from one homozygous Grey individual: ID2, and two heterozygous Grey individuals: ID3 and ID4.

\section{Targeted resequencing}

Two individuals were expressly chosen for massively parallel resequencing of the region surrounding the Grey mutation locus. One was a Lipizzaner horse (ID: L147) homozygous for Grey (Grey sample). The other was a non-Grey chestnut Arabian horse (ID: 800), with the closely related haplotype on which the Grey mutation occurred (i.e. "ancestral" non-Grey sample). In addition, five other non-Grey horses were included in the experiment: an Appaloosa, an Icelandic, a Knabstrupper, a Noriker and a Quarter Horse. The samples were prepared for sequencing as follows. Genomic DNA was fragmented and purified on QIAquick columns (Qiagen). Fragment ends were blunted and 5' phosphorylated, and a 3' overhang of a single adenosine was added. Illumina adapters (Illumina, San Diego, CA, USA) were ligated to the DNA fragments, which were subsequently hybridized to NimbleGen Sequence Capture Arrays (Roche NimbleGen, Madison, WI, USA), following manufacturer's recommendations. The arrays were designed to target the $352 \mathrm{~kb}$ region on horse chromosome 25, harbouring the haplotype previously associated to Grey. Following enrichment for target fragments on the arrays, Illumina adapter ligated fragments were amplified with adapter specific primers using a high-fidelity polymerase (Phusion, Finnzymes, Espoo, Finland) and purified on QIAquick columns (Qiagen). The enrichment-factor for each sample was assessed by quantitative PCR comparison to the same samples prior to hybridization. The enriched samples were then sequenced with Illumina Genome Analyzer (Illumina), obtaining single-end $35 \mathrm{bp}$ reads. Mapping of the reads and SNP calling were performed with the standard parameters of the MAQ program, version 0.6.6 [24].

\section{Genotyping}

The inferred genotypes for 10 selected SNPs were confirmed by PCR followed by Sanger sequencing of three individuals; Twilight, the thoroughbred mare that the genome sequence is derived from is heterozygous for Grey [17], an homozygous Grey horse (ID: L032) and the individual with a non-Grey haplotype closely related to Grey (ID: 800). Subsequently, TaqMan SNP Genotyping Assays were designed for five SNPs being either polymorphic in the Grey individual or not showing linkage disequilibrium with Grey (Figure 3 and Additional file 3). SNPs polymorphic among non-Grey individuals were not considered for further analysis, since we aimed at identifying other mutations associated with the Grey phenotype. In total, 357 Grey and non-Grey horses from 8 different breeds were genotyped for the selected SNPs in order to investigate if any of these SNPs could be associated with the Grey phenotype. Four Connemara horses with the late greying phenotype were included among these 357 horses to investigate possible additional mutations in the Grey haplotype. Samples were genotyped on an ABI7900HT instrument using the TaqMan allelic discrimination assay according to the manufacturer's instructions (Applied Biosystems). Each SNP was genotyped twice for every individual. The SNPs in repetitive regions were amplified by PCR for informative individuals and genotyped by Sanger sequencing (Additional file 3).

The Grey mutation was genotyped with long range PCR as previously described [2]. Since this method requires amplification of fragments $>4 \mathrm{~kb}$, the DNA isolated from hair samples or old blood samples of late greying Connemara horses could not be used. Therefore, their Grey genotypes were deduced from the TaqMan Copy Number Assay.

\section{Identification of a transposed fragment originating from STX17 3'UTR}

To identify the approximate size of a duplicated fragment corresponding to the sequence in the STX17 3'UTR that could explain the results from the TaqMan SNP screen, PCR products from two individuals genotyped as homozygous $C / C$, two individuals genotyped as $C / T$ but with a $C / T$-ratio of 3:1 and two individuals genotyped as $C / T$ with a $C / T$-ratio of 2:2 were amplified 5, 4, 3, 2 and $1 \mathrm{~kb}$ upstream and downstream of SNP2 (Additional file 3) using the Expand Long Template PCR system Mix 1 (Roche Diagnostics GmbH, Mannheim, Germany), in $25 \mu \mathrm{l}$ reactions using $125 \mathrm{ng}$ of genomic DNA. The PCR products were treated with ExoI and calf intestine alkaline phosphatase (CIAP) in ExoI buffer (all 
from Fermentas, St. Leon-Rot, Germany) at $85^{\circ} \mathrm{C}$ for $60 \mathrm{~min}$ to remove free nucleotides and excess primers prior to sequencing. The SNP was then genotyped by sequencing with primers flanking the polymorphism (Additional file 3). Sequences were analyzed and screened for mutations with Codon Code Aligner (CodonCode Corporation, Dedham, MA, USA).

\section{Additional files}

\section{Additional file 1: Lack of copy number variation outside and over the $5^{\prime}$ breakpoint of the STX17 duplication in constitutional DNA from Grey horses. 94 Grey Lipizzaner horses and one calibrator sample with a known copy number of 2 were included in the analysis. OUT = outside the duplicated sequence and BP $\mathrm{n}=$ the border between the 5 'flanking sequence and the 5'end of the duplicated sequence. Error bars represent the copy number range from the CopyCaller ${ }^{\text {TM }}$ Software analysis of quadruplicates in each assay.}

Additional file 2: The late greying Connemara horses are heterozygous for the STX17 duplication. Copy number assay for the STX17 duplication from tests with four different probes using constitutional DNA from the late greying Connemara horses SP1, SP2, SP3. The sample denoted 'Calibr' is a g/g individual with a known copy number of 2, used as a calibrator in the IN, OUT and BP $n$ analyses. Constitutional DNA from one $G / g$ and one $G / G$ horse was tested in the assay and the results are shown as a reference for the copy number expected from each genotype. The $G / G$ reference sample was used as a calibrator in the BP $\mathrm{d}$ analysis. IN = inside the duplicated sequence, OUT = outside the duplicated sequence, BP $n=$ the border between the $5^{\prime}$ flanking sequence and the $5^{\prime}$ end of the duplicated sequence and $\mathrm{BP} d=$ over the duplication breakpoint. Error bars represent the copy number range from the CopyCaller ${ }^{\text {TM }}$ Software analysis of quadruplicates in each assay.

Additional file 3: Primer sequences used for characterization of the region harbouring the Grey mutation in horses.

\section{Competing interests}

A patent has been filed concerning commercial applications of the diagnostic test for the Grey mutation. GRP, AG and LA are inventors on this patent application.

\section{Authors' contributions}

ES participated in the design of the study, carried out the Copy Number Assays, genotyped SNPs and Grey phenotypes, identified the transposed fragment and wrote the manuscript. Fl carried out the targeted resequencing and analyzed the results. SM, IC, MHS and JS contributed with material. CW, SS, KLT took part in the targeted resequencing. GRP contributed to the experimental design of the Copy Number Assay. AG took part in the analysis of tumour samples. LA supervised the study, participated in its design and coordination and helped to draft the manuscript. All authors read and approved the final manuscript.

\section{Acknowledgements}

We are grateful to Michael O. Hottiger, Institute of Veterinary Biochemistry, University of Zürich for kindly providing the equine melanoma cell lines, and to Jenny Hagenblad for information about late greying Connemara horses. The project received support from the Swedish Cancer Society, the Foundation for Strategic Research and the National Human Genome Research Institute. K.L.-T. is the recipient of a European Young Investigator award funded by the European Science Foundation.

\section{Author details}

${ }^{1}$ Department of Animal Breeding and Genetics, Swedish University of Agricultural Sciences, Box 597, SE-751 24 Uppsala, Sweden. ${ }^{2}$ Science for Life Laboratory Uppsala, Department of Medical Biochemistry and Microbiology, Uppsala University, Box 582, SE-751 23 Uppsala, Sweden. ${ }^{3}$ Department of Animal Breeding and Genetics, Swedish University of Agricultural Sciences,
Box 7023, SE-750 07 Uppsala, Sweden. ${ }^{4}$ Broad Institute of Harvard and MIT, 7 Cambridge Center, Cambridge, MA 02142 , USA. ${ }^{5}$ Center for Human Genetic Research, Massachusetts General Hospital, Boston, MA 02114 USA. ${ }^{6}$ Faculty of Veterinary Sciences, University of Sydney, Sydney, New South Wales 2006, Australia. ${ }^{7}$ Animal Science Department, Faculty of Agriculture, University of Zagreb, Zagreb HR-10000 Croatia. ${ }^{8}$ Clinical Department of Small Animal Surgery, University of Veterinary Medicine, Vienna, Austria. ${ }^{9}$ Department of Sustainable Agricultural Systems, University of Natural Resources and Applied Life Sciences, Vienna, A-1180 Vienna, Austria.

Received: 16 January 2012 Accepted: 18 July 2012

Published: 2 August 2012

\section{References}

1. Lerner AB, Cage GW: Melanomas in horses. Yale J Biol Med 1973, 46(5):646-649.

2. Rosengren Pielberg G, Golovko A, Sundström E, Curik I, Lennartsson J, Seltenhammer MH, Druml T, Binns M, Fitzsimmons C, Lindgren G, et al A cis-acting regulatory mutation causes premature hair graying and susceptibility to melanoma in the horse. Nat Genet 2008, 40(8):1004-1009.

3. Sutton RH, Coleman GT: Melanoma and the Graying Horse. In RIRDC Research Paper Series. Australia: Barton; 1997:1-34.

4. Fleury C, Berard F, Leblond A, Faure C, Ganem N, Thomas L: The study of cutaneous melanomas in Camargue-type gray-skinned horses (2): epidemiological survey. Pigment Cell Res 2000, 13(1):47-51.

5. Pielberg G, Mikko S, Sandberg K, Andersson L: Comparative linkage mapping of the Grey coat colour gene in horses. Anim Genet 2005, 36(5):390-395.

6. Feuk L, Carson AR, Scherer SW: Structural variation in the human genome. Nat Rev Genet 2006, 7(2):85-97.

7. Wright D, Boije H, Meadows JR, Bed'hom B, Gourichon D, Vieaud A, TixierBoichard M, Rubin CJ, Imsland F, Hallbook F, et al: Copy number variation in intron 1 of SOX 5 causes the Pea-comb phenotype in chickens. PLoS Genet 2009, 5(6):e1000512. e1000512.

8. Johansson Moller M, Chaudhary R, Hellmen E, Hoyheim B, Chowdhary B, Andersson L: Pigs with the dominant white coat color phenotype carry a duplication of the KIT gene encoding the mast/stem cell growth factor receptor. Mamm Genome 1996, 7(11):822-830.

9. Giuffra E, Tornsten A, Marklund S, Bongcam-Rudloff E, Chardon P, Kijas JM, Anderson SI, Archibald AL, Andersson L: A large duplication associated with dominant white color in pigs originated by homologous recombination between LINE elements flanking KIT. Mamm Genome 2002, 13(10):569-577.

10. Pielberg G, Olsson C, Syvanen AC, Andersson L: Unexpectedly high allelic diversity at the KIT locus causing dominant white color in the domestic pig. Genetics 2002, 160(1):305-311.

11. Gault J, Hopkins J, Berger R, Drebing C, Logel J, Walton C, Short M, Vianzon R, Olincy A, Ross RG, et al: Comparison of polymorphisms in the alpha7 nicotinic receptor gene and its partial duplication in schizophrenic and control subjects. Am J Med Genet B Neuropsychiatr Genet 2003, 123B(1):39-49.

12. Traherne JA: Human MHC architecture and evolution: implications for disease association studies. Int J Immunogenet 2008, 35(3):179-192.

13. Shlien A, Malkin D: Copy number variations and cancer susceptibility. Curr Opin Oncol 2010, 22(1):55-63.

14. Weir BA, Woo MS, Getz G, Perner S, Ding L, Beroukhim R, Lin WM, Province $M A$, Kraja A, Johnson $L A$, et al: Characterizing the cancer genome in lung adenocarcinoma. Nature 2007, 450(7171):893-898.

15. Mullighan CG, Goorha S, Radtke I, Miller CB, Coustan-Smith E, Dalton JD, Girtman K, Mathew S, Ma J, Pounds SB, et al: Genome-wide analysis of genetic alterations in acute lymphoblastic leukaemia. Nature 2007 446(7137):758-764.

16. Bastian $B C$, Olshen $A B$, LeBoit PE, Pinkel D: Classifying melanocytic tumors based on DNA copy number changes. Am J Pathol 2003, 163(5):1765-1770.

17. Wade CM, Giulotto E, Sigurdsson S, Zoli M, Gnerre S, Imsland F, Lear TL, Adelson DL, Bailey E, Bellone RR, et al: Genome sequence, comparative analysis, and population genetics of the domestic horse. Science 2009, 326(5954):865-867.

18. Bailey JA, Gu Z, Clark RA, Reinert K, Samonte RV, Schwartz S, Adams MD, Myers EW, Li PW, Eichler EE: Recent segmental duplications in the human genome. Science 2002, 297(5583):1003-1007. 
19. Chapman SW, Metzger N, Grest P, Feige K, von Rechenberg B, Auer JA, Hottiger MO: Isolation, establishment, and characterization of ex vivo equine melanoma cell cultures. In Vitro Cell Dev Biol Anim 2009, 45(3-4):152-162

20. Ben-Izhak O, Bar-Chana M, Sussman L, Dobiner V, Sandbank J, Cagnano M, Cohen H, Sabo E: Ki67 antigen and PCNA proliferation markers predict survival in anorectal malignant melanoma. Histopathology 2002, 41(6):519-525.

21. Sundström E, Komisarczuk AZ, Jiang L, Golovko A, Navratilova P, Rinkwitz S, Becker TS, Andersson L: Identification of a melanocyte-specific, microphthalmia-associated transcription factor-dependent regulatory element in the intronic duplication causing hair greying and melanoma in horses. Pigment Cell Melanoma Res 2012, 25(1):28-36.

22. Wong GK, Liu B, Wang J, Zhang Y, Yang X, Zhang Z, Meng Q, Zhou J, Li D, Zhang J, et al: A genetic variation map for chicken with 2.8 million single-nucleotide polymorphisms. Nature 2004, 432(7018):717-722.

23. Cao Y, Bryan TM, Reddel RR: Increased copy number of the TERT and TERC telomerase subunit genes in cancer cells. Cancer Sci 2008, 99(6):1092-1099.

24. Li H, Ruan J, Durbin R: Mapping short DNA sequencing reads and calling variants using mapping quality scores. Genome Res 2008, 18(11):1851-1858.

25. Mariat D, Taourit S, Guerin G: A mutation in the MATP gene causes the cream coat colour in the horse. Genet Sel Evol 2003, 35(1):119-133.

doi:10.1186/1471-2164-13-365

Cite this article as: Sundström et al:: Copy number expansion of the STX17 duplication in melanoma tissue from Grey horses. BMC Genomics 2012 13:365.

\section{Submit your next manuscript to BioMed Central and take full advantage of:}

- Convenient online submission

- Thorough peer review

- No space constraints or color figure charges

- Immediate publication on acceptance

- Inclusion in PubMed, CAS, Scopus and Google Scholar

- Research which is freely available for redistribution 